\title{
Dosimetric study of RapidArc plans with flattened beam (FB) and flattening filter-free (FFF) beam for localized prostate cancer based on physical indices
}

\author{
Birendra K Rout ${ }^{1,2}$, Kanaparthy R Muralidhar', Mirza Ali', Mukka C Shekar², Alok Kumar ${ }^{3}$ \\ ${ }^{1}$ Department of Radiation Physics, American Oncology Institute, Hyderabad, India. \\ ${ }^{2}$ Department of Physics, Jawaharlal Nehru Technological University, Hyderabad, India. \\ ${ }^{3}$ Department of Radiation Physics, Mahavir Cancer Sansthan, Patna, India.
}

Received July 09, 2014; Revised August 05, 2014; Accepted August 08, 2014; Published Online September 05, 2014

\section{Original Article}

\begin{abstract}
Purpose: To identify the continual diversity between flattening photon beam (FB) and Flattening Filter Free (FFF) photon beams for localized prostate cancer; and to determine potential benefits and drawbacks of using unflattened beam for this type of treatment. Methods: Eight prostate cases including seminal vesicles selected for this study. The primary planning target volume (PTVP) and boost planning target volume ( $\mathrm{PTV}_{\mathrm{B}}$ ) were contoured. The total prescription dose was $78 \mathrm{~Gy}$ (56 Gy to PTVP and an additional 22 Gy to PTVB). For all cases, treatment plans using 6MV with FB and FFF beams with identical dose-volume constraints, arc angles and number of arcs were developed. The dose volume histograms for both techniques were compared for primary target volume and critical structures. Results: A low Sigma index (FFF: $1.65+0.361$; FB: $1.725+0.39$ ) indicating improved dose homogeneity in FFF beam. Conformity index (FFF: $0.994+0.01$; FB: $0.993+0.01$ ) is comparable for both techniques. Minimal difference of Organ at risk mean dose was observed. Normal tissue integral dose in FB plan resulted $1.5 \%$ lower than FFF plan. All the plans displayed significant increase (1.18 times for PTVP and 1.11 for PTBв) in the average number of necessary MU with FFF beam. Conclusion: Diversity between FB and FFF beam plans were found. FFF beam accelerator has been utilized to develop clinically acceptable Rapid Arc treatment plans for prostate cancer with $6 \mathrm{MV}$.
\end{abstract}

Keywords: Flattening Filter-Free; Flattened Beam; Sigma-Index; Conformity Index; Normal Tissue Integral Dose; Rapid Arc

\section{Introduction}

Cancer is the major cause of leading deaths in the $21^{\text {st }}$ century in world with 14.1 million cases and 8.2 million deaths occurred in 2012. ${ }^{1}$ Among them, prostate cancer stands as important due to risk of secondary malignancies associated with intensity modulation radiation therapy (IMRT) with conventional 3-Dimentional conformal radiotherapy (3D-CRT). ${ }^{2}$ Among the different technologies adopted, to cure the prostate cancer; External radiotherapy is recognized as one of the important treatment option. ${ }^{3-4}$ The technology aims to destroy cancer cells by minimal damaging (due to risk of sec-

Corresponding author: Birendra Kumar Rout; Department of Radiation Physics, American Oncology Institute, Hyderabad, India.

Cite this article as:

Rout BK, Muralidhar KR, Ali M, Shekar MC, Kumar A. Dosimetric study of RapidArc plans with flattened beam $(\mathrm{FB})$ and flattening filter-free (FFF) beam for localized prostate cancer based on physical indices. Int J Cancer Ther Oncol 2014; 2(4):02046. DOI: $10.14319 /$ ijcto.0204.6 ondary malignancies) to the surrounding normal tissues. It creates a best possible balance between maximizing dose to prostate cancer cells and minimization of side effects. Intensity-modulated radiotherapy (IMRT) technology for localized intermediate risk prostate tumor/cancer allows less toxicity in comparison to $3 \mathrm{D}-\mathrm{CRT}$. $^{5}$

Among the external beam radiotherapy techniques; Rapid Arc (Varian Medical Systems, Palo Alto) is a volumetric radiotherapy technology that delivers a fast and precise sculpted 3D dose distribution with a single 360-degree rotation of the linear accelerator gantry to improve the standard of care and treatments. ${ }^{6}$ It is made possible by a treatment planning algorithm that simultaneously changes 3 -parameters during treatment by a) tuning rotation speed of the gantry; b) shape of the treatment aperture using the movement of multileaf collimator leaves; and c) delivery dose rate. Volumetric modulated arc therapy differs from existing 
techniques like helical IMRT or intensity-modulated arc therapy (IMAT) because it delivers dose to the whole volume, rather than segment by segment. The treatment planning algorithm ensures the treatment precision, helping to spare normal healthy tissue. ${ }^{7}$ Rapid arc, represented by the True beam $\mathrm{ST}_{\mathrm{x}}$ linear accelerator (Varian Medical Systems, Palo Alto) is an alternative volumetric modulated arc therapy technique. The treatment machine True beam $\mathrm{ST}_{\mathrm{x}}$, which is capability of wide spectrum of conformal treatment techniques like conformal beam static conformal arc, dynamic conformal arc, multiple choice IMRT and is capability of delivery of both flatten beam (FB) and flattening filter free (FFF) beams. ${ }^{8}$

Traditionally, the flattening filter in the X-ray beam path of a linear accelerator produce an almost uniform fluence over a collimated field. The removal of flattening filter leads to radially decreasing fluence distribution and thus to non-homogeneous dose distribution. Its positive influence on the peripheral dose has an advantage of reduced head scatter and MLC leakage, as well as a considerable increasing in the dose rate, which has a beneficial effect on modern therapy methods. ${ }^{9}$ To improve shielding in the treatment head; Hall et al. suggested the use of secondary jaws to track the MLC and removal of the flattening filter as a source of scattered radiation in IMRT. The disadvantage of non-uniform, conical fluence distribution could be taken into account with intensity modulation radiation therapy (IMRT) in the optimization algorithm.

The present study aims to identify the main and continual diversity between flattened photon beam (FB) and Flattening Filter Free (FFF) photon beams using Eclipse treatment planning system and True Beam $\mathrm{ST}_{\mathrm{x}}$ linear accelerator for localized prostate cancer. Subsequently; the study explores the potential benefits for determining the possibility to develop clinically acceptable Rapid Arc treatment plans for prostate cancer with $6 \mathrm{MV}$. This may reduce secondary malignancies without sacrificing local control or increasing toxicity rates. ${ }^{10}$

\section{Methods and Materials}

A total of eight patients have been selected for treatment with external beam radiation therapy for localized prostate cancer analysis. This study includes prostate with seminal vesicles as target. Computed Tomography (CT) images were acquired with an empty rectum and full bladder for all patients and imported to the Eclipse treatment planning system, version 10.0.26 (Varian Medical Systems, Palo Alto, CA) and registered. The attending radiation oncologist manually segmented prostate and seminal vesicles based on CT images.
The primary planning target volume (PTVP) was defined to include $0.7 \mathrm{~cm}$ margin around the prostate and seminal vesicles in all directions except the posterior direction, where 0.5 $\mathrm{cm}$ margin was added. The boost planning target volume (PTV $\left.{ }_{\mathrm{B}}\right)$ was defined to include $0.7 \mathrm{~cm}$ margin around the prostate in all direction except the posterior; where $0.5 \mathrm{~cm}$ margin was added. All of the prostate patients at our institute were treated with image-guidance; based on measured localization uncertainties such as inter-user reproducibility and intra-fraction motion. ${ }^{11}$ Rectum, Bladder, Bowel and left and right femoral head were contoured as Organ At Risk(OARs) based on CT images. Both FB and FFF beams were developed for each patient in the eclipse treatment planning system using $6 \mathrm{MV}$ photons in a Varian made True Beam ST $\mathrm{x}$ mchine; having dynamic capability of 120 high definition MLC. The anisotropic analytical algorithm (AAA, version 10.0.26) has been used. ${ }^{12-13}$ Dose grid sizes of $(3 \times 3 \times 3) \mathrm{mm}^{3}$ were used throughout the study. The slice thickness of the CT images used for planning purpose was $3 \mathrm{~mm}$. The total prescription dose was $78 \mathrm{~Gy}$ in 39 fractions. The prescription dose of the primary plans was $56 \mathrm{~Gy}$ to PTV $\mathrm{P}$ and prescription dose of the boost plans was $22 \mathrm{~Gy}$ to $\mathrm{PTV}_{\mathrm{B}}$ following the Radiation Therapy Oncology Group (RTOG) 0521 protocol.

A primary plan for PTVP and separate plan for PTV generated using FB and FFF beam for each patient. All the patients' image sets were chosen in such way that, there was not much variation in anatomy. All the patients' anterior-posterior (AP) and lateral separations were very close. The average AP diameter was $21 \mathrm{~cm}$ and the lateral diameter was $32 \mathrm{~cm}$. The average volume of PTV and $\mathrm{PTV}_{\text {в were } 197.68 \mathrm{cc}}$ (range, 169.8-239.3 cc) and $147.66 \mathrm{cc}$ (range, $101.2 \mathrm{cc}-166.9$ $\mathrm{cc}$ ) respectively. The average volume of bladder, rectum, right femoral head, left femoral head and Bowel were $211.36 \mathrm{cc}$ (range, $64.6 \mathrm{cc}-371.7 \mathrm{cc}$ ), $79.93 \mathrm{cc}$ (range, $55.5 \mathrm{cc}-114.5 \mathrm{cc}$ ), 164.34 cc (range, 141.9 cc-211.5 cc), 163.95 cc (range, 141.2 cc-212.5 cc) and 1243.9 cc (range, 380 cc-1680 cc) respectively. Two arcs $\left(182^{\circ}\right.$ to $178^{\circ} ; 178^{\circ}$ to $\left.182^{\circ}\right)$ were used in both clock wise and anti-clock wise directions for all patients in treatment planning. For the clock wise arc, the collimator was rotated $20^{\circ}$ while for the anti-clockwise arc; the collimated was rotated $340^{\circ}$ in order to reduce the effect due to inter-leaf leakage. All plans were generated using Eclipse planning system (version 10.0.26) and the volumetric dose optimization method followed the same systematic strategy regarding the objective and priorities. The optimization constraints for all plans are listed in Table 1. These constraints and weightings were set initially and then modified by either relaxing or tightening during the optimization process based on the real-time updated dose-volume histograms (DVHs) of structures. Figure 1 is showing a transversal view of rapid arc plan setup using two arcs and the $\mathrm{PTV}_{\mathrm{p}}$. 


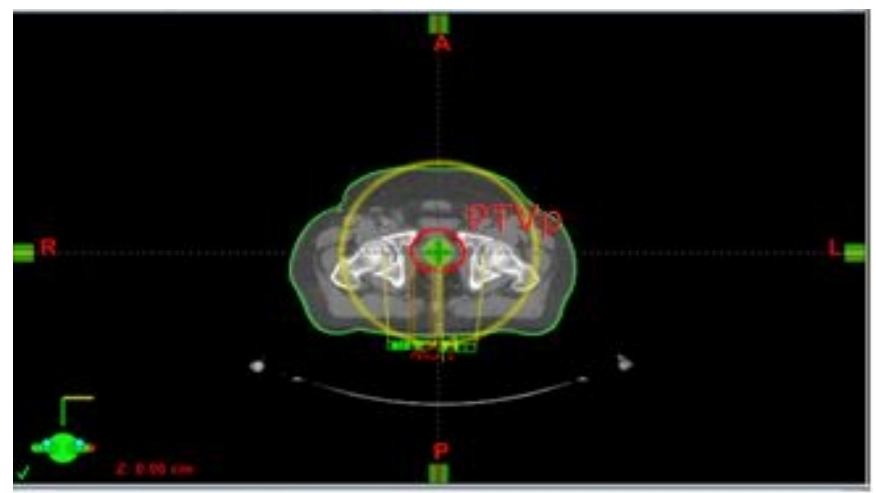

FIG. 1: A transversal view of rapid arc plan setup using two Arcs and the Primary target volume (PTV

Treatment plan was done based on the radiotherapy objective of delivering a therapeutic dose to a well-defined target while minimizing dose to the surrounding normal tissue and critical organs. It requires optimization of conformity of the prescription dose to the planning target volume, dose homogeneity within the PTV and dose to the surrounding normal tissue and critical organs. The following parameters were evaluated for comparing cumulative dose volume histogram of the patient such as Sigma-index, conformity index, mean doses to bladder, rectum, bowel, right femoral head and left femoral head.

The better representation of homogeneity could be defined in term of sigma index (S-index). ${ }^{14}$ This uses the differential $\mathrm{DVH}$, unlike other indices, which is cumulative DVH. $\mathrm{S}$-index is a measure of the standard deviation of the doses about the mean dose. The S-index is expressed as follow.

$$
\mathrm{S}-\text { index }=\mathrm{D}_{\mathrm{SD}}=\sum \sqrt{\left(\mathrm{D}_{\mathrm{i}}-\mathrm{D}_{\text {mean }}\right)} \times \frac{\mathrm{v}_{\mathrm{i}}}{\mathrm{V}}
$$

$D_{\text {mean }}$ is the mean dose of the target (PTV in this study) curve. $\mathrm{D}_{\mathrm{i}}$ is dose to the $\mathrm{i}^{\text {th }}$ bin having a volume $\mathrm{vi}$. $\mathrm{V}=$ total volume of the target.

The conformity index (CI) was defined to compare the treatment plans. The conformity index is defined as the ratio of the $95 \%$ isodose volume divided by the PTV that is enclosed by the $95 \%$ of the isodose line. From this definition, as the conformity index approaches 1 , the more conformal is the treatment plan.

\section{Dose distribution, PTV coverage and organs at risk.}

In addition to providing information on the homogeneity of radiation doses; DVHs can also be used to assess target coverage index, defined as the percentage of the tumor volume that received the prescribed dose. Ideally; tumor DVH would be a step function, with $100 \%$ of the target receiving the exact prescribed dose. However, actual DVH curves are not step functions, because of constraints imposed by tumor volume and other organs at risk (OAR). Rules for PTV was set such that the prescribed dose covered at least $95 \%$ of the PTV (D95) and the PTV volume receiving $>107 \%$ of the prescription was limited to $2 \%$ in line with ICRU report 50 and ICRU report 62. To achieve this objective, a constraint for $\mathrm{D}_{100}$ was set to receive $\geq 98 \%$ of prescription and constraint for maximum dose $\left(D_{\max }\right)$ was set to receive $\leq 102 \%$ of the prescription in the optimization process for both plans. For all of the cases, treatment plans using $6 \mathrm{MV}$ with maximum dose rate of 600 $\mathrm{MU} / \mathrm{min}$ for FB beams and 1,400 MU/min for FFF beams were developed under identical conditions such as dose-volume constraints, arc angle and number of arcs.

The volumes that received a minimum of $50 \mathrm{~Gy}, 60 \mathrm{~Gy}, 65$ Gy, 70 Gy, 75 Gy (V50Gy, V60Gy, V65Gy, V70Gy, V75Gy) for rectum, 65 Gy, 70 Gy, 75 Gy, 80 Gy (V65Gy, V70Gy, V75Gy, V80Gy) for bladder and $45 \mathrm{~Gy}, 15 \mathrm{~Gy}\left(\mathrm{~V}_{45 \mathrm{~Gy}}, \mathrm{~V}_{15 \mathrm{~Gy}}\right)$ for bowel respectively, were selected to evaluate volumes of high dose. ${ }^{15-16}$

\section{Normal Tissue Integral Dose and MU}

The integral dose (in unit of liter-Gy) was defined as the absorbed dose integrated over the voxels in the entire volume excluding PTV. The integral dose (ID) (mean dose $\times$ tissue volume) received by normal tissue (NTID) was calculated from dose-volume histograms. For comparison, the integral dose ratio was obtained by dividing the integral dose from a FB plan by the integral dose from FFF plan. MU was analyzed for FFF and FB plans and reported.

\section{Statistical Analysis}

Statistical analysis was performed with Statistical Analysis Software (SAS), version 9.3. Mean values and standard deviation of the mean (SD) were collected. Relative dosimetric changes were compared applying the paired t-test. A two-sided $p$ value $\leq 0.05$ was considered statistically significant. Confidence intervals (CI) included $95 \%$ of the measured data.

\section{Results}

Sigma index (S-index) and Conformity index (CI) The S-index and conformity indices for all the prostate patients in the both FB and FFF beam with $6 \mathrm{MV}$ photons are summarized in Table 2-3. The relative efficacy (FB/FFF) 
values for, S-index and conformity index are 1.045.0.998 for PTVP and 1.113, 0.999 for PTV в respectively. The transversal view of comparative dose distribution between FB plan and FFF beam plans is shown in Figure 2.
Dose distribution, PTV coverage and organs at risk

Two typical dose-volume histograms for 6-MV plans, with and without a flattening filter, are compared in Figure 3-5. The PTV coverage was more uniform in the plans with unflattened beams.

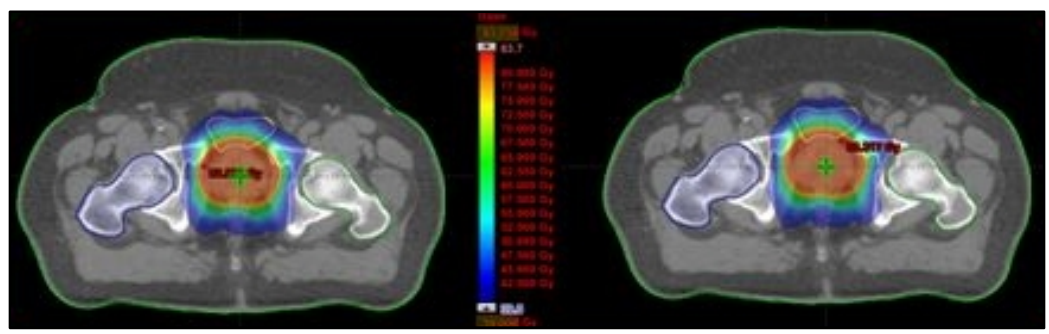

FIG. 2: Transversal view of comparative dose distribution between FB plan (Lt side) and FFF beam plans (Rt side). Here 100\% considered 78Gy (composite plan).

TABLE 1: Dose-Volume constraints and relative weightings used for optimization of both FB and FFF Rapid Arc plans.

\begin{tabular}{|c|c|c|}
\hline PTVs and OARs & Dose-Volume constraints & Relative weighting \\
\hline PTVP and PTV & $D_{100} \geq 98 \% ; D_{\max } \leq 102 \%$ & $110-120$ \\
\hline Rectum and Bladder & $\begin{array}{l}V_{70} \leq 30 \% ; V_{50 \%} \leq 50 \% ; V_{30} \leq 70 \% ; \text { D5-D10 } \\
\text { of } 60-70 G y ; D_{\max } \leq 100 \%\end{array}$ & $60-80$ \\
\hline Bowel & $\begin{array}{l}V_{50 \%} \leq 30 \% ; V_{30 \%} \leq 50 \% ; V_{10 \%} \leq 70 \% \\
\text { Dmax } \leq \text { total of } 45 \mathrm{~Gy}\end{array}$ & $50-60$ \\
\hline Femoral heads & $V_{20 \%}-V_{30 \%} \leq 30 \%$; $D_{\max \leq t o t a l}$ of $45 \mathrm{~Gy}$ & $50-60$ \\
\hline
\end{tabular}

TABLE 2: S- index and conformity index of PTVP plans for FB and FFF beam.

\begin{tabular}{ccccc}
\hline \hline & \multicolumn{2}{c}{ FB } & \multicolumn{2}{c}{ FFF beam } \\
\hline Pt.No. & S-index & CI & S-index & CI \\
\hline 1 & 1.9 & 0.991 & 1.8 & 0.992 \\
2 & 2.4 & 0.97 & 2.4 & 0.971 \\
3 & 1.9 & 0.998 & 1.8 & 0.999 \\
4 & 1.7 & 0.999 & 1.7 & 0.999 \\
5 & 1.9 & 0.987 & 1.6 & 0.993 \\
6 & 1.7 & 0.999 & 1.4 & 0.999 \\
7 & 1.2 & .0999 & 1.4 & 0.998 \\
8 & 1.1 & 0.999 & 1.1 & 0.999 \\
Mean \pm SD & $1.725 \pm 0.39$ & $0.993 \pm 0.01$ & $1.65 \pm 0.361$ & $0.994 \pm 0.01$ \\
\hline \hline
\end{tabular}

TABLE 3: Sigma indices and conformity index of PTVв plans for FB and FFF beam.

\begin{tabular}{cccccc}
\hline \hline & \multicolumn{2}{c}{ FB } & \multicolumn{2}{c}{ FFF beam } \\
\hline Pt.No. & S-index & CI & S-index & CI \\
\hline 1 & 1.9 & 0.992 & 1.8 & 0.993 \\
2 & 2.4 & 0.98 & 2.4 & 0.981 \\
3 & 1.6 & 0.998 & 1.6 & 0.998 \\
4 & 1.7 & 0.992 & 1.7 & 0.993 \\
5 & 1.7 & 0.995 & 1.3 & 0.998 \\
6 & 1.5 & 0.999 & 1.2 & 1 \\
7 & 1.9 & 0.998 & 1.3 & 0.998 \\
8 & 1 & 0.999 & 1 & 0.999 \\
Mean \pm SD & $1.713 \pm 0.372$ & $0.994 \pm 0.006$ & $1.538 \pm 0.413$ & $0.995 \pm 0.006$ \\
\hline \hline
\end{tabular}


TABLE 4: Mean dose statistics (with SD) of Organ at risk for composite plan.

\begin{tabular}{|c|c|c|c|c|c|}
\hline \multirow[t]{2}{*}{ Structure } & \multicolumn{2}{|c|}{$\begin{array}{c}\text { Dose-Volume } \\
\text { constraints }\end{array}$} & \multicolumn{3}{|c|}{ Volume achieved in \% } \\
\hline & & & $\mathrm{FB}$ & FFF & $\mathrm{P}$ \\
\hline \multirow[t]{5}{*}{ Rectum } & $V_{50 G y}$ & $<50 \%$ & $29.82 \pm 5.9 \%$ & $28.62 \pm 4.4 \%$ & 0.239 \\
\hline & V60Gy & $<35 \%$ & $20.08 \pm 4.1 \%$ & $19.35 \pm 3.0 \%$ & 0.244 \\
\hline & V65Gy & $<25 \%$ & $16.04 \pm 3.3 \%$ & $15.65 \pm 2.6 \%$ & 0.382 \\
\hline & V70Gy & $<20 \%$ & $12.4 \pm 2.9 \%$ & $12.2 \pm 2.4 \%$ & 0.538 \\
\hline & V75Gy & $<15 \%$ & $8.42 \pm 2.8 \%$ & $8.52 \pm 2.4 \%$ & 0.633 \\
\hline \multirow[t]{4}{*}{ Bladder } & $V_{65 G y}$ & $<50 \%$ & $19.26 \pm 10.2 \%$ & $18.17 \pm 9.4 \%$ & 0.425 \\
\hline & V70Gy & $<35 \%$ & $14.84 \pm 7.4 \%$ & $15.02 \pm 7.7 \%$ & 0.135 \\
\hline & V75Gy & $<25 \%$ & $11.74 \pm 5.6 \%$ & $11.9 \pm 5.8 \%$ & 0.301 \\
\hline & V80Gy & $<15 \%$ & $3.36 \pm 3.97 \%$ & $3.50 \pm 3.4 \%$ & 0.736 \\
\hline \multirow[t]{2}{*}{ Bowel } & $\mathrm{V}_{45 \mathrm{~Gy}}$ & $<195 \mathrm{cc}$ & $0.48 \pm 1.08 \%$ & $0.48 \pm 1.09 \%$ & 0.801 \\
\hline & V15Gy & $<120 \mathrm{cc}$ & $4.79 \pm 7.72 \%$ & $4.73 \pm 7.62 \%$ & 0.276 \\
\hline Rt Femoral Head & V50Gy & $<5 \%$ & $0.0 \pm 0.0 \%$ & $0.0 \pm 0.0 \%$ & - \\
\hline Lt Femoral Head & V50Gy & $<5 \%$ & $0.0 \pm 0.0 \%$ & $0.0 \pm 0.0 \%$ & - \\
\hline
\end{tabular}

TABLE 5: Average Maximum dose statistics (with SD) and average dosimetric indices (With SD) of PTV and Organ at risk for composite plan.

\begin{tabular}{lcccccc}
\hline Structure & \multicolumn{3}{c}{ Mean Dose in Gy } & \multicolumn{3}{c}{ Maximum Dose in Gy } \\
& FB & FFF & P & FB & FFF & p \\
\hline PTV & $79.56 \pm 0.82$ & $79.64 \pm 0.72$ & 0.303 & $82.48 \pm 0.94$ & $82.38 \pm 1.17$ & 0.675 \\
Rectum & $37.46 \pm 4.75$ & $37.46 \pm 4.59$ & 1.000 & $80.99 \pm 1.46$ & $81.18 \pm 1.28$ & 0.417 \\
Bladder & $37.7 \pm 11.86$ & $37.9 \pm 12.02$ & 0.203 & $81.99 \pm 0.56$ & $81.69 \pm 0.66$ & 0.873 \\
Bowel & $3.0 \pm 2.89$ & $3.08 \pm 2.84$ & 0.047 & $30.7 \pm 24.2$ & $30.3 \pm 23.6$ & 0.301 \\
RT FH & $15.71 \pm 1.88$ & $15.96 \pm 1.82$ & 0.083 & $35.09 \pm 4.44$ & $34.86 \pm 5.13$ & 0.467 \\
LT FH & $15.94 \pm 1.85$ & $16.04 \pm 1.97$ & 0.170 & $37.38 \pm 4.39$ & $37.29 \pm 4.52$ & 0.531 \\
\hline \hline
\end{tabular}

For the rectum; there was no dose differences between the two treatment modalities in the volume exposed to $60 \mathrm{~Gy}, 65$ Gy, 70 Gy and 75 Gy. Only small difference was observed for the volume exposed to $50 \mathrm{~Gy}$ which is not statistically significant ( $\mathrm{p}=0.239$ ). In case of bladde $r$, there were no dose differences for the volume exposed to $70 \mathrm{~Gy}, 75 \mathrm{~Gy}$ and $80 \mathrm{~Gy}$ but small difference was noted for the volume exposed to 65 Gy, which is statistically insignificant $(p=0.424)$. For the bowel; there was no significant dose differences between the two treatment modalities in the volume exposed to $45 \mathrm{~Gy}$ or $15 \mathrm{~Gy}$ and femoral heads were within the required constraints for all patients. Table 4 shows mean dose statistics with standard deviation of organ at risk for composite plan.

Table 5 shows average maximum dose statistics (with SD) and average dosimetric indices with standard deviation of PTV and organ at risk for composite plan. No significant differences were noted between FB and FFF beam plans in the average doses delivered to the bladder, rectum, and femoral heads. A significant increase in mean dose of $2.6 \%$ ( $<<0.047$, $95 \% \mathrm{CI}=0.001$ to 0.149 ) to the bowel was measured for FFF beam plans compared with FB plans. The average maximum dose of PTV is $105.74 \%$ and $105.62 \%$ of prescribe dose for both FB and FFF beam plans respectively. No significant differences between two modalities were observed with respect to mean average maximum dose to bladder, rectum, femoral heads and bowel.

\section{Normal tissue integral dose and MU}

Because of radiation could induce second malignancies, ${ }^{2}$ integral dose was used as an index to compare techniques for radiation induced second cancers. The NTID (mean \pm SD) was $125.1 \pm 23.57$ liter-Gy for FB plan and 126.98 \pm 24.14 liter-Gy for FFF beam. FFF beam delivered $1.50 \%$ more dose compared with the FB beam $(\mathrm{p}<0.002,95 \% \mathrm{CI}=-2.775$ to -0.985). Comparison of the integral dose for FB and FFF beam in Rapid Arc composite plans is shown in Figure 6. For flattened beam-intensity modulation treatments, including IMRT and Rapid Arc, the number of MU needed are an indicator for the mean body dose. ${ }^{17} \mathrm{We}$ found that MU (mean \pm SD) was $675 \pm 36.66$ for FB plans and $796 \pm 88.11$ for FFF beam plans in PTV. MU (mean \pm SD) was $615 \pm 80.29$ for FB plans and $683 \pm 94.34$ for FFF plans in PTVв. Compared with FB plan, the MU significantly increased by $17.9 \%(\mathrm{p}<0.003$, 95\% CI $=-188.3$ to -54.44$)$ in PTVP and $11.05 \%(\mathrm{p}<0.004$, 95\% CI $=-103.9$ to -33.087 ) in PTV в for FFF beam plan.

\section{Discussion}

These Indices clearly represent more homogeneous dose for FFF beam plan than for the FB plans. The measured data found is in accordance with reported values by Yoon et al. The lower the value of S-index indicates the better is the homogeneity (Table 2-3). From our data between FFF and FB plans, FFF beam plans are showing lower S-indices for both 
PTVР and РTVв plans indicating improved dose homogeneity compared to FB plans.

Miften et al. ${ }^{18}$ have demonstrated the use of target conformity index (TCI) and normal tissue-sparing index (NTSI) to assist in the process of judging the merit of a clinical treatment plan. But, in this work, the widely accepted conformity index was used to evaluate the conformity of the treatment plans (Table 2-3) for all the patients using both techniques. The mean conformity index was 0.993 \& 0.994 for PTVP and 0.994 \& 0.995 for PTV в (FB \& FFF) beam plans respectively. These small differences indicate that these plans are nearly identical in their conformity of dose to the target.
PTV coverage for prostate cancer. Depending on the beam energy, 6MV FFF beam produced superior IMRT plans than $6 \mathrm{MV} \mathrm{FB} .{ }^{20}$ However, plans and treatment delivery parameters were not fully optimized for FFF beams. The improvement of PTV coverage for either plan might have been possible with different user-specific cost functions for both the PTV and organ at risk been reported. A negligible difference in PTV coverage between flattened and FFF beams IMRT prostate plans has been observed by Stathakis et al. ${ }^{21} \mathrm{~A}$ similar results have been reported by Zwahlen et al; ${ }^{22}$ using 6MV hypo fractionated volumetric modulated arc therapy with and without flattening filter got similar results regarding PTV coverage for prostate cancer.

Vassiliev et al. ${ }^{19}$ reported in their planning study with and without flattening filter, similar treatment plans regarding

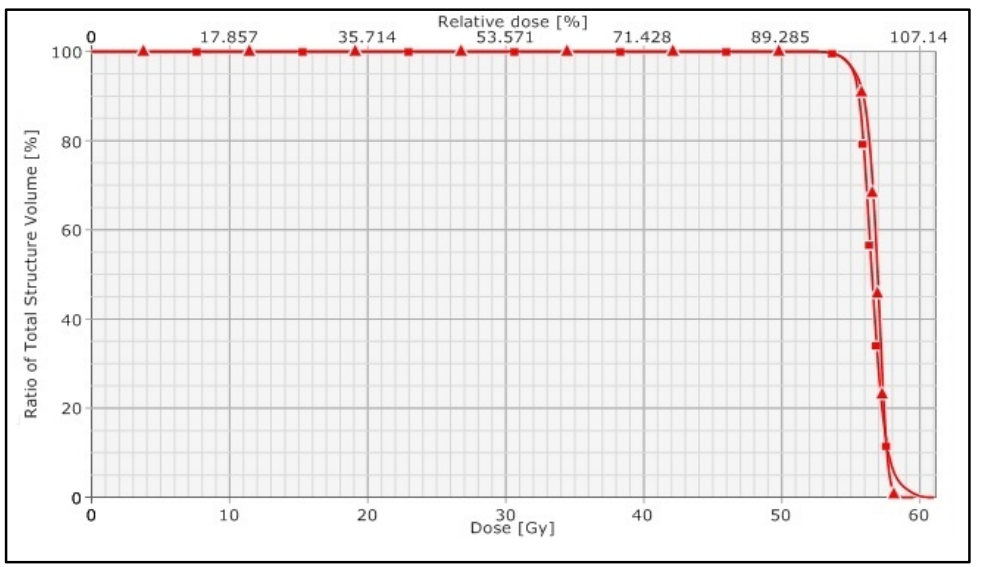

FIG. 3: Comparison of the dose -volume histograms for FB (square) and FFF beam (triangle) for PTVP Rapid Arc plan.

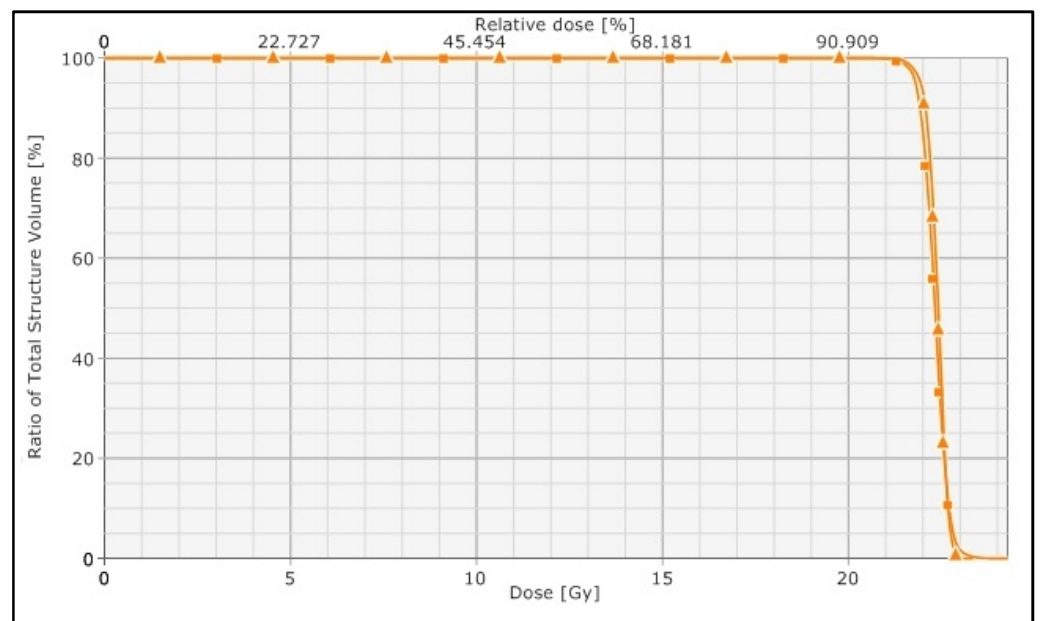

FIG. 4: Comparision of the dose -volume histograms for FB (square) and FFF beam (triangle) for PTV в Rapid Arc plan. 


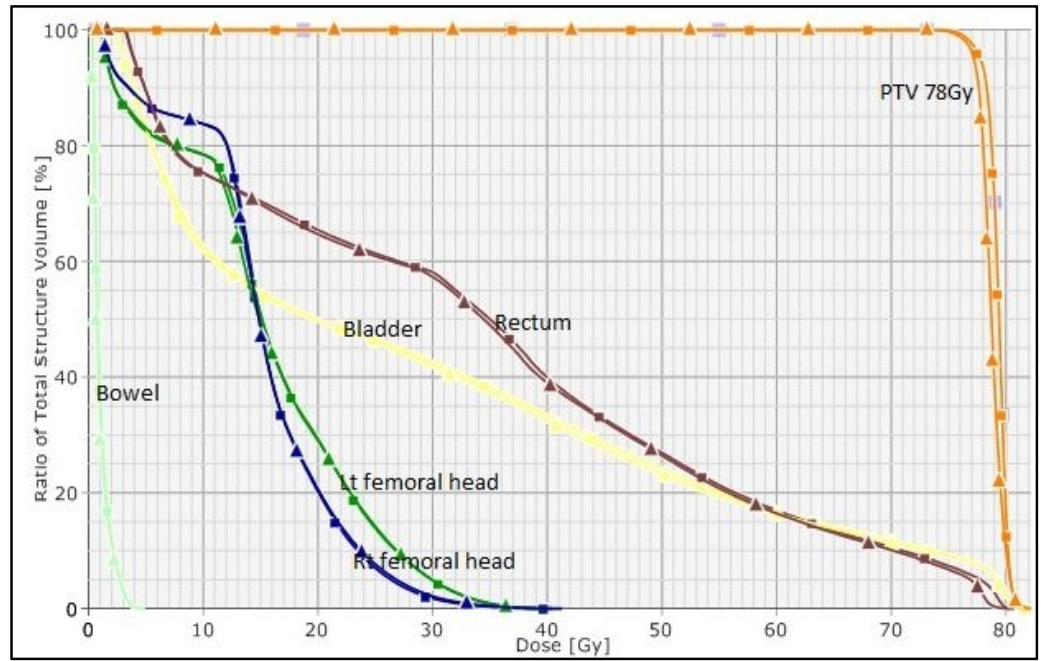

FIG. 5: Comparision of the dose -volume histograms for FB (triangle) and FFF beam (square) for PTV78Gy and OARs in Rapid Arc composite plan.

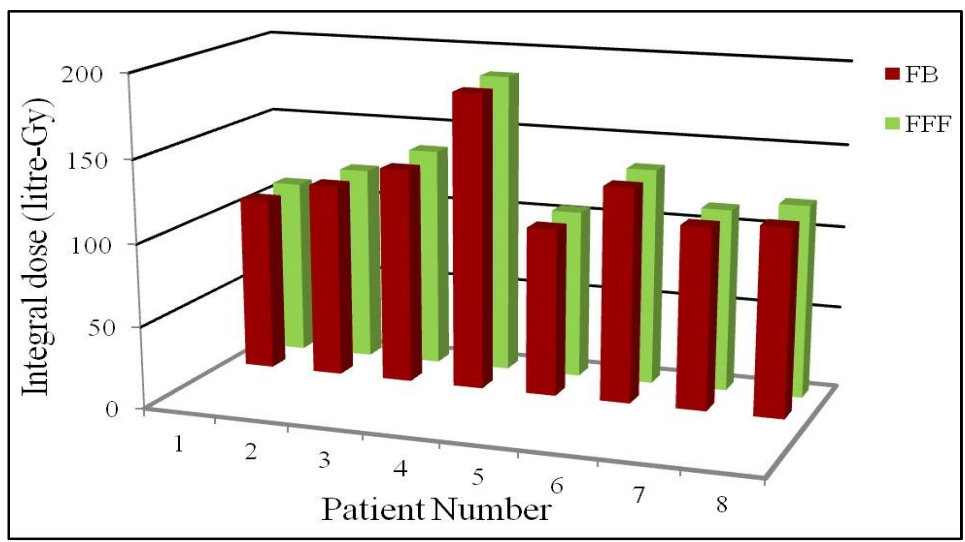

FIG. 6: Comparison of the integral dose for FB and FFF beam in Rapid Arc composite plans.

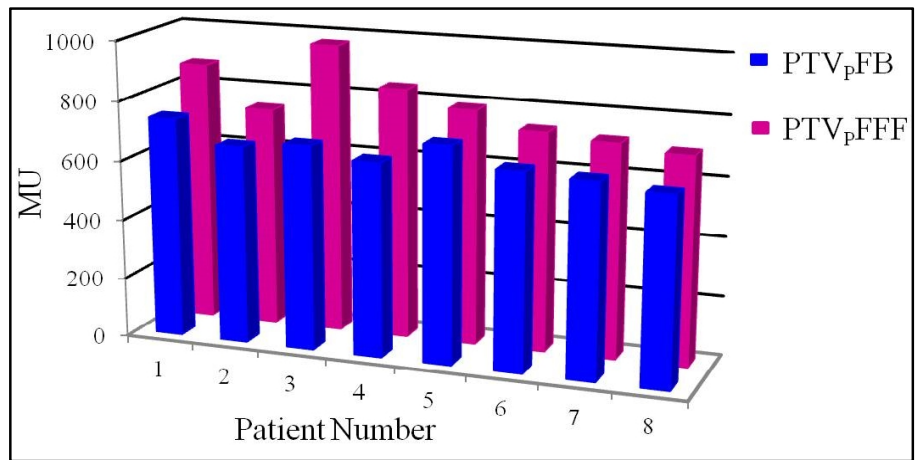

FIG. 7: Comparison of the MUs for FB and FFF beam for PTVP Rapid Arc plans. PTVP FB is the primary planning treatment volume Flattened Beam plans and PTVP FFF is the primary planning treatment volume Flattening Filter-Free beam plans. 


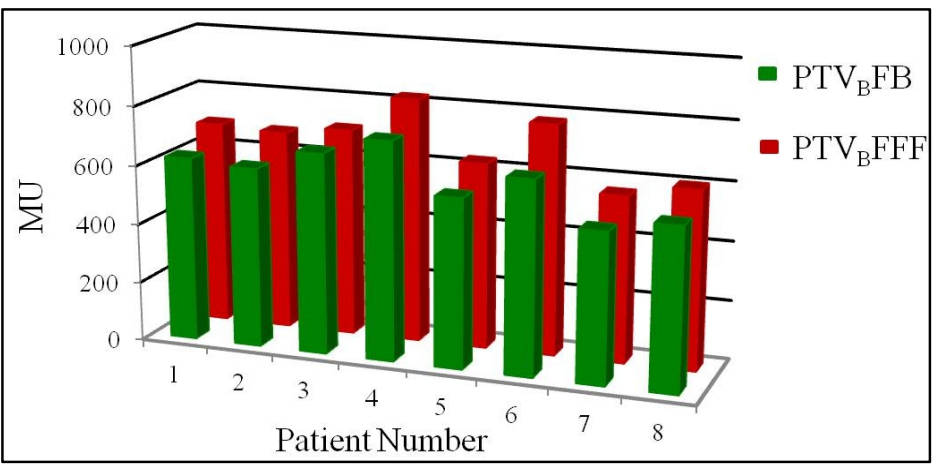

FIG. 8: Comparison of the MUs for FB and FFF beam for PTV в Rapid Arc plans. PTV plans and $\mathrm{PTV}_{\mathrm{B}}$ FFF is the Boost planning treatment volume Flattening Filter-Free beam plans.

The increased NTID can be attributed to the softer photon spectrum and resultant higher skin dose from FFF beams. Therefore, it does not necessarily indicate higher doses are required to internal organs. Moreover, it is possible to reduce the skin dose by increasing beam energy. ${ }^{23}$

The average number of necessary MU with the use of FFF beams is shown in Figure 7-8. Because of larger treatment fields, the dose uniformity within an irradiated treatment field will need to be "modulated" by MLC movements (IMRT) to cut down the higher beam intensity near the central portion of the FFF X-ray beam. Thus, larger MUs are required compared to a conventional (flattened) X-ray beam. MLC movements (IMRT) are now being used to flatten the FFF $\mathrm{X}$-rays to provide dose uniformity within those large PTVs. The high dose rates from the FFF X-rays are now being off-set by the larger MUs requirements. Zwahlen et al. reported similar results as we found here. In contrary, Vassiliev et al. and Stathakis et al. found a significant decrease in the number of MU for their FFF treatment plans. The reason was that our FFF beams were calibrated in order that $100 \mathrm{MU}$ corresponded to $1 \mathrm{~Gy}$ at the depth of maximum dose, as this was common for flattened beam. ${ }^{24}$

However, for all prototype LINACs with FFF capabilities used by other groups, only the flattened beams were calibrated. This resulted in a higher dose per $100 \mathrm{MU}$ for the unflattened beams. Therefore they report a decrease in number of MU that we could not confirm in our study. Our data demonstrated that the mean body dose slightly decreased with the mean energy of the beam, despite the increase in number of MU that did not affect the mean body dose. From our study; we did not found beam on time difference between these techniques. The time advantage increased when using higher dose per fraction. However, was insignificant for standard fractionation of $2 \mathrm{~Gy} .{ }^{25}$

\section{Conclusion}

For treatment plans with Rapid Arc, FFF beams resulted in dose distribution similar to flattened beams. No significant differences were found between FFF and FB plans in the mean dose and maximum dose of bladder and rectum. Bowel and femoral heads being at a clinically significant distance from PTVs (Prostate and seminal vesicles) usually experience small doses. Sigma indices are lower, which correspond to lower HI in FFF beam plans, indicating improved dose homogeneity for both PTVP and $\mathrm{PTV}_{\mathrm{B}}$. No dose constraints violation occurred for both the techniques. Plans with FFF beams tend to have comparable conformity and higher NTID than plans with FB beams. The increased NTID can be attributed to the softer photon spectrum and resultant higher skin dose from FFF beams. Therefore, it does not necessarily indicate higher skin doses to internal organs. Though the differences were insignificant, using a FFF beam accelerator; ${ }^{26}$ it has been possible to develop clinically acceptable Rapid Arc treatment plans for prostate cancer with $6 \mathrm{MV}$.

\section{Conflict of interest}

The authors declare that they have no conflicts of interest. The authors alone are responsible for the content and writing of the paper.

\section{References}

1. Ferlay J, Soerjomataram I, Ervik M, et al GLOBOCAN 2012 v1.0, Cancer Incidence and Mortality Worldwide: IARC Cancer Base No. 11 2013. Lyon, France: International Agency for Research on Cancer. Available from http://globocan.iarc.fr

2. Hall EJ. Intensity-modulated radiation therapy, protons, and the risk of second cancers. Int J Radiat Oncol Biol Phys 2006; 65:1-7.

3. Nguyen PL, Gu X, Lipsitz SR, et al. Cost implications of the rapid adoption of newer technologies 
for treating prostate cancer. J Clin Oncol 2011; 29:1517-24.

4. Rukstalis DB. Treatment options after failure of radiation therapy-a review. Rev Urol 2002; 4 Suppl 2:S12-7.

5. Heidenreich A, Bolla M. Joniau S, et al. Guidelines on Prostate Cancer; European Association of Urology 2011

6. Yoo S, Wu QJ, Lee WR, Yin FF. Radiotherapy treatment plans with RapidArc for prostate cancer involving seminal vesicles and lymph nodes. Int $J$ Radiat Oncol Biol Phys 2010; 76:935-42.

7. Seppala J, Suilamo S, Kulmala J, et al. A dosimetric phantom study of dose accuracy and build-up effects using IMRT and RapidArc in stereotactic irradiation of lung tumours. Radiat Oncol 2012; 7:79.

8. Wiant DB, Terrell JA, Maurer JM, Yount CL, Sintay BJ. Commissioning and validation of Brain$\mathrm{LAB}$ cones for $6 \mathrm{X} \mathrm{FFF}$ and 10X FFF beams on a Varian TrueBeam STx. J Appl Clin Med Phys 2013;14:4493.

9. Georg D, Knoos T, McClean B. Current status and future perspective of flattening filter free photon beams. Med Phys 2011; 38:1280-93.

10. Sun M, Ma L. Treatment Exceptionally large prostate cancer patients with low energy intensity modulated photons. J Appl Clin Med Phys 2006; 7:43-9.

11. Orton NP, Tome WA. The impact of daily shifts on prostate IMRT dose distribution. Med Phys 2004; 31:1-4.

12. Bragg CM, Windate $\mathrm{K}$, Conway J. Clinical implementations of the anisotropic analytical algorithm for IMRT treatment planning and verification. Radiother Oncol 2008; 86:276-84.

13. Lu, L. Dose calculation algorithms in external beam photon radiation therapy. Int J Cancer Ther Oncol 2013; 1:01025.

14. Yoon M, Park SY, Shin D, et al. A new homogeneity index based on statistical analysis of the dose-volume histogram. J Appl Clin Med Phys 2007; 8:9-17.

15. Lawrence BM, Ellen DY, ANDREW J, et al. Use of normal tissue complication probability model in the clinic. Int J Radiat Oncol Biol Phys 2010; 76: S10-S19.

16. Michalski JM, Purdy JA, Winter K, et al. Preliminary report of toxicity following $3 \mathrm{D}$ radiation therapy for prostate cancer on 3DOG/RTOG 9406. Int J Radiat Oncol Bio Phys 2000; 46:391-402.

17. Followill D, Geis P, Boyer A. Estimates of whole-body dose equivalent produced by beam intensity modulated conformal therapy. Int J Radiat Oncol Biol Phys 1997; 38:667-72.

18. Miften MM, Das SK, Su M, Marks LB. A dose-volume based tool for evaluating and ranking
IMRT treatment plans. J Appl Clin Med Phys 2004; 5:1-14.

19. Vassiliev ON, Titt U, Ponisch F, et al. Dosimetric properties of photon beams from a flattening filter free clinical accelerator. Phys Med Biol 2006; 51:1907-17.

20. Vassiliev ON, Kry SF, Kuban DA, et al. Treatment-planning study of prostate cancer intensity-modulated radiotherapy with a Varian clinic operated without a flattening filter. Int $J$ Radiat Oncol Biol Phys 2007; 68:1567-71.

21. Stathakis S, Esquivel C, Gutierrez A, et al. Treatment planning and delivery of IMRT using 6and $18 \mathrm{MV}$ photon beams without flattening filter. Appl Radiat Isot 2009; 67:1628-37.

22. Zwahlen DR, Lang S, Hrbacek J, et al. The use of photon beams of a flattening filter-free linear accelerator for hypofractionated volumetric modulated arc therapy in localized prostate cancer. Int $J$ Radiat Oncol Biol Phys 2012; 83:1655-60.

23. Aoyama H, Westerly DC, Mackie TR, et al. Integral radiation dose to normal structures with conformal external beam radiation. Int I Radiant Oncol Biol Phys 2006; 64:962-967.

24. Almond PR, Biggs PJ, Coursey BM, et al. AAPM's TG-51 protocol for clinical reference dosimetry of high-energy photon and electron beams. Med Phys 1999; 26:1847-70.

25. Fu W, Dai J, Hu Y, et al. Delivery time comparison for Intensity-modulated radiation therapy with/ without flattening filter: A planning Study. Phys Med Biol 2004; 49:1535-47.

26. Lang S, Reggiori G, Puxeu Vaquee J, et al. Pretreatment quality assurance of flattening filter free beams on 224 patients for intensity modulated plans; a multicentric study. Med Phys 2012; 39:1351-6. 